\title{
Investigation of Electron-Positron Correlations by Monte Carlo Simulation
}

\author{
E. BorońsKI \\ W. Trzebiatowski Institute for Low Temperature and Structure Research \\ Polish Academy of Sciences, P.O. Box 1410, 50-950 Wrocław 2, Poland \\ Earlier theoretical studies, as well as recent calculations of positron anni- \\ hilation rates in metals, show that some basic problems concerning electron- \\ positron $(\mathrm{e}-\mathrm{p})$ interaction have not been solved satisfactorily, even for ho- \\ mogeneous media (the electron gas). In turn, the "computer experiments" \\ e.g. quantum Monte Carlo simulations applied to these problems by several \\ authors yielded only fragmentary, inaccurate and even incorrect results for \\ smaller electron densities. It is shown in the present paper that the quantum \\ Monte Carlo method may be useful in investigations of positron interactions \\ with electrons. Reasonable annihilation rates have been obtained owing to \\ appropriate construction of the trial function and taking into account the \\ 3-particle correlations (i.e. dependence of the electron-electron (e-e) inter- \\ action on the distance from the positron). Moreover, the method of "exact \\ determination" (without any fitting) of positron annihilation rates on the \\ basis of the variational trial function was proposed. One also found the way \\ of calculating the momentum dependent enhancement factors, the quantities \\ not achievable within the Monte Carlo method until now.
}

PACS numbers: 71.60.+z, 78.70.Bj, 02.70.Ss

\section{Introduction}

The theory of positron annihilation in metals can already help to a great extent in interpretation of experimental results. However, some unexplained discrepancies between the theory and experiment, even for simple metals, manifest an incomplete understanding of electron-positron interaction in condensed matter $[1,2]$. For example, still it is not clear why the annihilation rates for simple metals resulting from the most advanced theories (e.g. [3-5]) are markedly higher than the corresponding experimental values (cf. also Ref. [6]). In turn, the theories based on more simplified assumptions [7] (cf. [8]) fit the experiment better and 
the difference between results of different theories for e.g. $r_{\mathrm{s}}=2$ or $r_{\mathrm{s}}=3$ exceeds $10 \%$. Therefore one should turn back to the essentials and study the interesting properties of $\mathrm{e}-\mathrm{p}$ interactions on the basis of the positron+electron gas model.

It seems that at present, owing to the progress in computer technology, the quantum Monte Carlo (QMC) method could be a useful tool for solving some problems concerning many-body interactions $[9,10]$. However, the known realizations of this method applied to electron-positron systems were not able to yield satisfactory results for positron annihilation rates and gave no advice how to get momentum dependent enhancement factors. The both mentioned quantities are very important and directly correspond to experimental data.

The QMC studies of the positron in an electron gas have been performed by Ortiz, Gilgien, and Fraser, however, the results have been presented only in their Ph.D. theses [11-13]. Their calculations concerning the annihilation rate, that is the quantity directly referring to the experiment, are unfortunately quite inaccurate, becoming even unreasonable for $r_{\mathrm{s}}>4$ (they fall down below $2 \times 10^{9} \mathrm{~s}^{-1}$ (Fig. 1)).

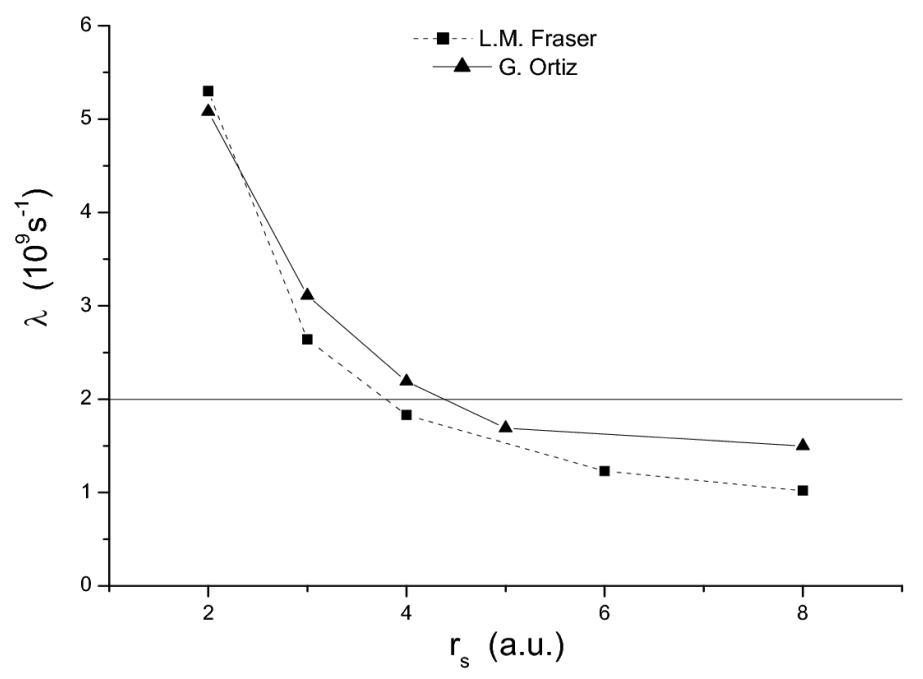

Fig. 1. Positron-electron annihilation rates according to Ortiz [11] and Fraser [13].

The present paper shows that the mentioned deficiencies of the Monte Carlo method can be removed and reasonable values of positron annihilation rates in the electron gas are obtainable. Preliminary calculations have been performed for several values of the electron densities. They indicate what modifications of the many-body wave function are necessary. Some details of the method have been presented earlier on PSPA-34 [14]. 


\section{The method}

\subsection{The variational quantum Monte Carlo (VQMC) method}

The total Hamiltonian $H$ (in atomic units) for the system of $N$ electrons and 1 positron is

$$
H=-\frac{1}{2} \sum_{i}^{N} \nabla_{\boldsymbol{x}_{i}}^{2}-\frac{1}{2} \nabla_{\boldsymbol{x}_{+}}^{2}+\sum_{i<j}^{N} \frac{1}{\left|\boldsymbol{x}_{i}-\boldsymbol{x}_{j}\right|}-\sum_{i}^{N} \frac{1}{\left|\boldsymbol{x}_{i}-\boldsymbol{x}_{+}\right|}+\Lambda,
$$

where the indices $i$ and $j$ correspond to the electrons, + corresponds to the positron, and $\Lambda$ is a constant potential energy representing the positive background and ensuring charge neutrality. In order to simulate the infinite system one has to assume the periodic boundary conditions (a number $N$ of particles is contained in the simulation cell (like Wigner-Seitz cell) and this cell is repeated in all directions).

The trial wave function $\Psi$ is parameter-dependent and should resemble the true wave function $\Psi_{0}$. The parameters of $\Psi$ can be found by minimization of the total energy of the system given by the expectation value of the Hamiltonian, with respect to variational parameters. According to Monte Carlo method the expectation value of any operator can be evaluated by taking the average of local values of this operator over $M$ configurations $(M \rightarrow \infty)$.

The configurations $X_{i}$, where $X$ is a $3 N$-dimensional vector representing the positions of $N$ particles, are generated using the Metropolis algorithm.

The minimization method used in this work bases on a combination of analytical derivatives method [15] and stochastic gradient method [16].

\subsection{The $V Q M C$ trial function}

The positron-many electron trial wave function (the symbol $X$ denotes the set of electron coordinates $\left.X=\left\{\boldsymbol{x}_{1}, \boldsymbol{x}_{2}, \ldots \boldsymbol{x}_{N}\right\}\right)$ can be written as

$$
\Psi\left(\boldsymbol{x}_{1}, \boldsymbol{x}_{2}, \ldots \boldsymbol{x}_{N}, \boldsymbol{x}_{+}\right)=D_{\uparrow}\left(X^{\uparrow}\right) D_{\downarrow}\left(X^{\downarrow}\right) \varphi\left(\boldsymbol{x}_{+}\right) J(X),
$$

where $D_{\uparrow}\left(X^{\uparrow}\right)$ and $D_{\downarrow}\left(X^{\downarrow}\right)$ are Slater determinants (consisting of plane waves, for $N / 2$ spin-up $\uparrow$ and $N / 2$ spin-down $\downarrow$ electrons, respectively), $\varphi\left(\boldsymbol{x}_{+}\right)$is the positron wave function, and $J$ is a generalization of the Jastrow factor

$$
J=\prod_{i} \exp \left[v_{i+}\left(r_{i+}\right)\right] \cdot \prod_{i<j} \exp \left[v_{i j}\left(r_{i j}\right)\right] \cdot \prod_{i<j} \exp \left[v_{i j+}\left(r_{i j}, r_{i+}, r_{j+}\right)\right],
$$

where $r_{i j}=\left|\boldsymbol{x}_{i}-\boldsymbol{x}_{j}\right|, r_{i+}=\left|\boldsymbol{x}_{i}-\boldsymbol{x}_{+}\right|, \boldsymbol{x}_{i}$ and $\boldsymbol{x}_{+}$are the $i$-th electron and positron coordinates, respectively.

The general form of the functions $v$ is assumed (according to Ref. [17]) as the following:

$$
v_{i j}(r)=\bar{v}_{i j}(r)+v_{i j}^{1}(r),
$$


where $\bar{v}_{i j}(r)$ is

$$
\bar{v}_{i j}(r)= \begin{cases}\frac{a_{i j} r}{1+b_{i j} r}+s_{i j} & \text { if } \quad r \leq R, \\ p_{i j}(r) & \text { if } \quad R<r \leq \frac{\Omega^{1 / 3}}{2^{5 / 6}}, \\ 0 & \text { otherwise, }\end{cases}
$$

and where $p_{i j}(r)$ is a sixth order polynomial chosen so that $\bar{v}_{i j}(r)$ are twice continuously differentiable at both boundaries.

The constants $a_{i j}(r)$ are determined by the cusp condition [18] (the relation between the value of the pair-correlation function and its derivative at $r=0$ ), but the other parameters $\left(b_{i j}\right.$ and $\left.s_{i j}\right)$ are to be found (the coefficients in $p_{i j}(r)$ depend on $a_{i j}(r), b_{i j}$, and $\left.s_{i j}\right)$. They differ for the cases of interaction of the electron with the one of parallel or antiparallel spin or with the positron. The functions $v_{i j}^{1}(r)$ are assumed in Ref. [17] in a simple Gaussian form $v_{i j}^{1}(r)=A_{i j} \mathrm{e}^{-\alpha_{i j} r^{2}}\left(A_{i j}\right.$ and $\alpha_{i j}$ are variational parameters).

Below one proposes more complicated form of $v_{i j}^{1}(r)$. It is assumed on the basis of the comparison of figures for the variational and diffusion results for the electron-electron correlation functions in Ref. [17]. This new function can already in variational calculations correct to some extent the shape (and properties) of electron-electron correlation hole

$$
v_{i j}^{1}(r)=A_{i j}\left(1+c r^{2}\right) \mathrm{e}^{-\alpha r^{2}-\gamma r^{4}} \cos (d r), \quad r \equiv r_{i j} .
$$

This function does not change the cusp conditions, $c$ and $d$ are additional variational parameters, the constant $\gamma$ is chosen so that the function (and its derivatives) becomes practically zero at the boundary of the simulation cell.

A similar form of the correction $v_{i j}^{1}(r)$ can be applied to the electron-positron part of the Jastrow factor (the parameters are different).

Finally, the 3-particle correlation (electron-electron-positron) can be taken into account in the following way:

$$
v_{i j+}^{1}=A_{i j+}\left(\mathrm{e}^{-a r_{i+}^{2}}+\mathrm{e}^{-a r_{j+}^{2}}\right) \mathrm{e}^{-\gamma r_{i j}^{4}}\left(D+r_{i j}^{2} \mathrm{e}^{-b r_{i j}}\right),
$$

again, $A_{i j+}, a, b$, and $D$ are new, additional variational parameters.

The above function is applied only for the electrons of antiparallel spins. The form of the $r_{i j-}$ dependent part of the function $v_{i j+}^{1}$ is based on the approximate form of the difference between electron-electron pair correlation functions for different electron densities. The part depending on $x$ and $y$ is the function that yields the amplitude of this difference depending on the distance of the two considered electrons from the positron.

\subsection{Transformation of the coordinate system}

In order to determine the electron-positron pair-correlation function for the considered system it is convenient to introduce a new set of coordinates [19, 20, 14]: 


$$
R=\frac{1}{N+1}\left(\boldsymbol{x}_{+}+\sum_{i=1}^{N} \boldsymbol{x}_{i}\right), \quad \xi_{i}=\boldsymbol{x}_{i}-\boldsymbol{x}_{+},
$$

where $R$ is the coordinate of the center of mass of the system and $\xi_{i}$ are coordinates given by the distances between electrons and the positron.

Then the Hamiltonian of the system can be separated into two parts

$$
H=H_{0}+H^{\prime},
$$

where

$$
H_{0}=-\frac{1}{2(N+1)} \nabla_{R}^{2}
$$

and

$$
H^{\prime}=\sum_{i}^{N}\left(-\frac{1}{2 \mu} \nabla_{\xi_{i}}^{2}-\frac{1}{\left|\xi_{i}\right|}\right)+\sum_{i<j}^{N}\left(\frac{1}{2 \mu} \nabla_{\xi_{i}} \nabla_{\xi_{j}}+\frac{1}{\left|\xi_{i}-\xi_{j}\right|}\right)+\Lambda,
$$

where $\mu=\frac{1}{2}$.

\subsection{The pair correlation function}

The electron-positron correlation function $g(r)$ is the quantity directly connected with the positron annihilation rate. For the electron gas the annihilation rate $\lambda$ can be obtained from the formula

$$
\lambda\left(r_{\mathrm{s}}\right)=\frac{12}{r_{\mathrm{s}}^{3}} g\left(r_{\mathrm{s}}, 0\right) \times 10^{9} / \mathrm{s},
$$

where $g\left(r_{\mathrm{s}}, 0\right)$ is the contact density (the value of $g(r)$ at $r=0$ for a given electron density).

Conventionally, for the electron gas the methods of calculation of $g(r)$ within the MC are based on collecting, during the simulation process, the statistics of the number of particles whose distances from the positron drop into spherical layers of the volume $\Delta V_{r}=4 \pi r^{2} \Delta r$. Such methods of calculating e-e and e-p pair-correlation functions $g(r)$ have been used in Refs. [11-13]. If the number of such particles is $n_{r}$, then

$$
g(r) \sim \frac{n_{r}}{\Delta V_{r}} .
$$

This procedure yields the values that are greatly scattered, in particular in the neighbourhood of $r=0$ (since $\Delta V_{r} \rightarrow 0$ for $r \rightarrow 0$ there is no finite value for $r=0$ ). Therefore, one finds the $g(r)$ by fitting a function (cf. Ref. [16]) to the scattered values, requiring some additional assumptions. They, however, can change the $g(r)$ so it will not exactly correspond to the given simulation. 
Instead, we propose the following formula in the new coordinates (cf. [14]):

$$
g(\boldsymbol{r})=\lim _{M \rightarrow \infty} \frac{1}{M} \sum_{i}^{M} \frac{\frac{1}{N} \sum_{j}^{N}\left|\Psi\left(\xi_{1}^{i}, \xi_{2}^{i}, \ldots, \xi_{j}^{i}=\boldsymbol{r}, \ldots, \xi_{N}^{i}\right)\right|^{2}}{\left|\Psi\left(\xi_{1}^{i}, \xi_{2}^{i}, \ldots \xi_{N}^{i}\right)\right|^{2}}
$$

that gives $g(r)$ with the accuracy dependent only on the number $M$ of sampled (according to the Metropolis method) configurations of particles. $\xi_{j}^{i}$ means the coordinate $\xi$ of $j$-th particle in $i$-th configuration. The averaging in Eq. (14) with respect to the number $N$ of indistinguishable particles corrects additionally the statistics of the calculation.

The above formula yields, in particular, directly the value of contact density and no additional fitting is needed. The example of calculations according to Eqs. (13) and (14) is discussed in section Results.

\subsection{Enhancement factors}

The electrons in the system containing a single positron contribute in different ways to the value of the electron density on the positron. The knowledge of this effect is crucial for the interpretation of the experimental data.

The $2 \gamma$ annihilation rate to the particular final state $\Phi_{\nu}$ of $N-1$ electrons (with a hole of momentum $k$ ) and emitted gamma quanta with total momentum $q$ can be defined as

$$
\begin{aligned}
\Lambda_{\nu}(\boldsymbol{q}) & \sim \sum_{i=1}^{N} \mid \int \mathrm{d} \tau \Phi_{\nu}^{*}\left(\boldsymbol{x}_{1}, \boldsymbol{x}_{2}, \ldots, \boldsymbol{x}_{i-1}, \boldsymbol{x}_{i+1}, \ldots, \boldsymbol{x}_{N}\right) \frac{\mathrm{e}^{\mathrm{i} \boldsymbol{q} \boldsymbol{x}_{+}}}{2 \pi^{3 / 2}} \\
& \times\left. O_{i}^{\mathrm{s}} \delta\left(\boldsymbol{x}_{i}-\boldsymbol{x}_{+}\right) \Psi\left(\boldsymbol{x}_{1}, \boldsymbol{x}_{2}, \ldots, \boldsymbol{x}_{N}, \boldsymbol{x}_{+}\right)\right|^{2},
\end{aligned}
$$

where $O_{i}^{\mathrm{s}}$ is the spin projection operator and $\delta\left(\boldsymbol{x}_{i}-\boldsymbol{x}_{+}\right)$acts as the positron coordinate projection operator (see e.g. $[4,21]$ ).

If the many-body wave function is described by determinants then $\Phi_{\nu}$ can be given by a determinant of order $N-1$ (without $i$-th column and $k$-th row). Of course, the other determinant (with the opposite spin) remains unchanged.

Thus, the momentum-dependent enhancement factor $\varepsilon$ can be defined by using Eq. (15), given the proper normalization (by utilizing the calculated value of the contact density).

Defining $\Psi\left(\xi_{1}, \xi_{2}, \xi_{i}=0, \ldots, \xi_{N}\right) \equiv \Psi^{0}\left(R_{i}\right)$ and $\Phi_{k}^{*}\left(\xi_{1}^{j}, \xi_{2}^{j}, \ldots, \xi_{i-1}^{j}\right.$, $\left.\xi_{i+1}^{j} \ldots, \xi_{N}^{j}\right) \equiv \Phi_{k}^{*}\left(R_{i}\right)$, we get the following Monte Carlo formula [14]:

$$
\varepsilon(\boldsymbol{k})=S \frac{\frac{1}{M} \sum_{j}^{M} \frac{\left\langle\Phi_{k}^{*}\left(R_{i}^{j}\right) \Psi^{0}\left(R_{i}^{j}\right)\right\rangle_{i}}{\left|\Psi\left(R^{j}\right)\right|^{2}}}{\frac{1}{M} \sum_{j}^{M} \frac{\left\langle\left|\Phi_{k}^{*}\left(R_{i}^{j}\right)\right|^{2}\right\rangle_{i}}{\left|\Psi\left(R^{j}\right)\right|^{2}}} \times \frac{\frac{1}{M} \sum_{j}^{M} \frac{\left\langle\Phi_{k}^{*}\left(R_{i}^{j}\right) \Psi^{0}\left(R_{i}^{j}\right)\right\rangle_{i}}{\left|\Psi\left(R^{j}\right)\right|^{2}}}{\frac{1}{M} \sum_{j}^{M} \frac{\left\langle\left|\Psi^{0}\left(R_{i}^{j}\right)\right|^{2}\right\rangle_{i}}{\left|\Psi\left(R^{j}\right)\right|^{2}}}
$$

where the expressions $\left|\Phi_{k}^{*}\left(R_{i}^{j}\right)\right|^{2} /\left|\Psi\left(R_{i}\right)\right|^{2}$ and $\left|\Psi^{0}\left(R_{i}\right)\right|^{2} /\left|\Psi\left(R_{i}\right)\right|^{2}$ can be treated as weights in the integrals, the points $\xi$ are distributed according to $|\Psi|^{2}$. 
Thus, with our method we are able to calculate not only $g(\boldsymbol{r})$ but also $\varepsilon(\boldsymbol{k})$ in a single Monte Carlo run.

\section{Results}

The calculations have been performed for 226 electrons in the fcc-type elementary cell. In these preliminary calculations the chosen electron densities corresponded to $r_{\mathrm{s}}=1,2,5$, and 8 . At first, in the minimization procedure the trial function parameters have been found only for the system of electrons without the positron. For $r_{\mathrm{s}}=5$ and 8 this function has been corrected in relation to the Ortiz method by using Eq. (6). The next step was to add the positron to the system and find the appropriate parameters for the full electron-positron wave function, having the first set of parameters (corresponding only to the electrons) frozen. The whole number of parameters considered was 20. Successively, the appropriate corrections to e-p Jastrow factor and 3-particle correlations (7) were taken into account. Several tens thousands of iterations have been performed during the minimization procedure for each $r_{\mathrm{s}}$ value. In each iteration 12 new different configurations of the coordinates of all particles have been considered.

The parameters obtained have been used then to determine the electronpositron contact densities for $r_{\mathrm{s}}=2,5$, and 8 and the momentum dependent enhancement factors for $r_{\mathrm{s}}=2$. The contact densities were obtained according to Eq. (14). An example of the electron-positron correlation function obtained from the present calculations is shown in Fig. 2 (solid line). The scattered points visualize the results obtained conventionally (according to Eq. (13)). When applying the new method one does not need any fitting that can introduce an additional uncertainty (resulting from additional physical assumptions). For example, for

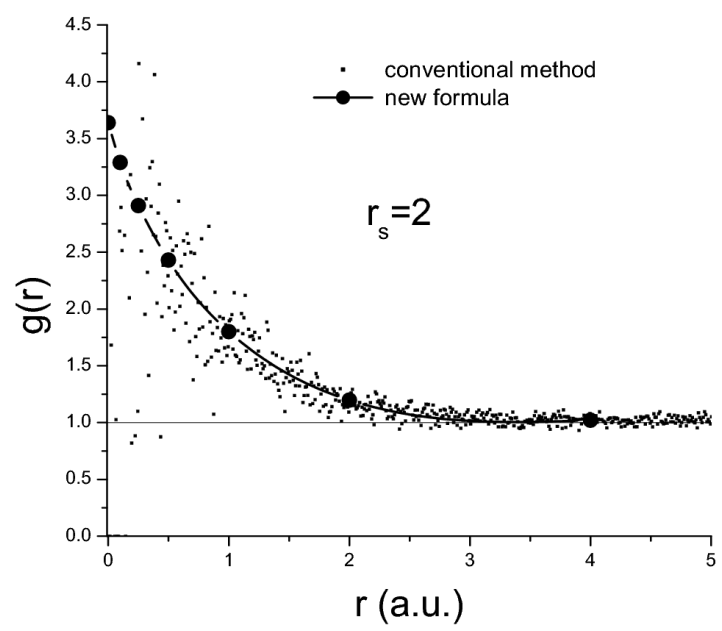

Fig. 2. Electron-positron correlation function $g(r)$ for $r_{\mathrm{s}}=2$. 
greater distances the correlation function oscillates and the period of these oscillations is not constant (it only converges to a constant in infinity). The character of these oscillations has some influence on the value of $g(r)$ in the cusp. This effect was not included in conventional fitting.

The parameters corresponding to $r_{\mathrm{s}}=2$ have also been used to calculate the momentum dependent enhancement factor. It is shown in Fig. 3. It resembles the corresponding figure of Kahana [22] (or Arponen and Pajanne [23]), in general. However, since the number of considered electrons is limited, only few $k$ points in the momentum space are available. Therefore, the line in the figure is not smooth.

The most important problem to solve was to find reasonable annihilation rates for lower electron densities. The attempts of three authors (Ortiz, Gilgien, and Fraser) in their Ph.D. theses turned out to be futile. Only the diffusion Monte

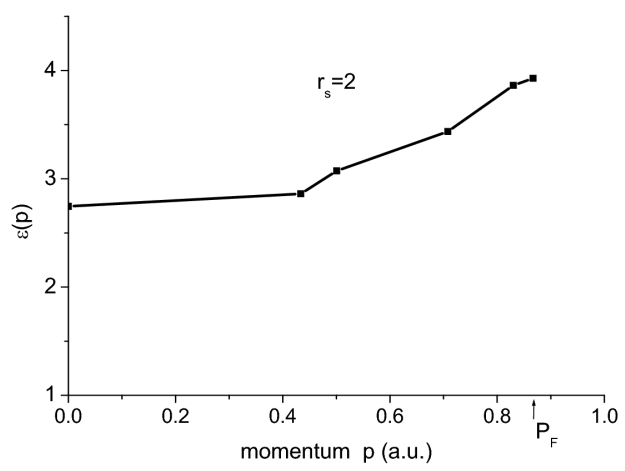

Fig. 3. Momentum dependent enhancement factor for $r_{\mathrm{s}}=2$.

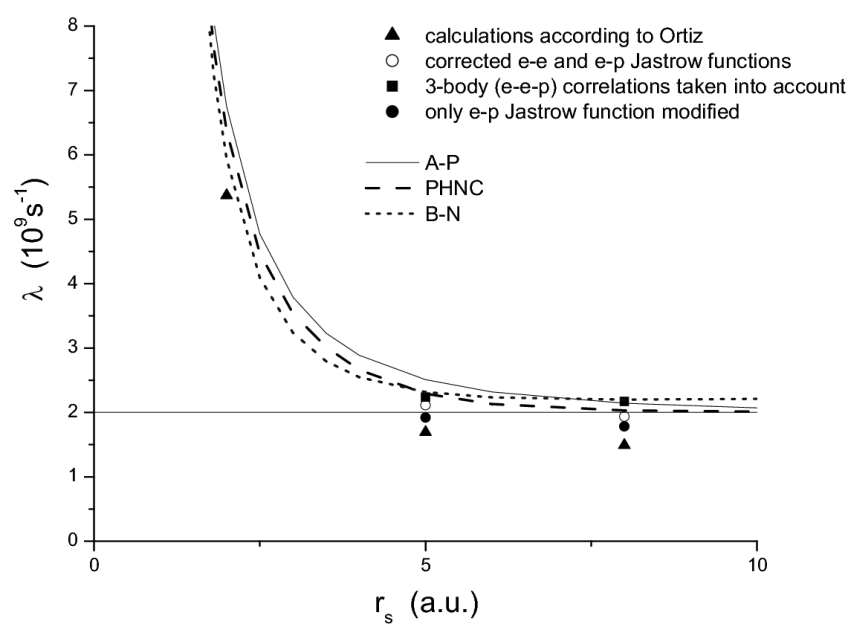

Fig. 4. Annihilation rates vs. $r_{\mathrm{s}}$ calculated in this work (symbols). The solid (A-P), dashed (PHNC, i.e. perturbed hypernetted chain), and dotted lines correspond to formulas for the $\mathrm{e}^{+}-\mathrm{e}^{-}$correlation function used in Refs. [3], [4], and [8] respectively. 
Carlo calculations of Gilgien seemed to be reasonable, however, she could not get even approximate values of corresponding contact densities, and finally did not publish the results.

The results of this work are presented in Fig. 4. The contribution of different modifications of the Jastrow factor is shown by using different symbols. It is worth noticing that already correction of purely electronic part of the Jastrow factor (that changes the shape of electron-electron correlation hole for homogeneous system of electrons) is very important. If one corrected only the electron-positron part of the Jastrow factor the improvement would not be sufficient. For $r_{\mathrm{s}}=5$ only both corrections lead to crossing the "barrier" $2 \times 10^{9} \mathrm{~s}^{-1}$. A considerable contribution (for $r_{\mathrm{s}} \geq 5$ ) comes from taking into account 3-particle correlations (Eq. (7)) (as pointed out long time ago by Rubaszek et al. [5]). In particular for $r_{\mathrm{s}}=8$ it exceeds $11 \%$ of the total value of the annihilation rate.

\section{Summary and conclusions}

It has been shown that quantum Monte Carlo method can be a useful tool in studies of such important quantities in positron physics as annihilation rates and momentum dependent enhancement factors. The first mentioned quantity can be calculated with arbitrary accuracy at the given theoretical assumptions. The drawbacks of hitherto existing VQMC approaches to positron in the homogeneous electron gas, such as much too low values of contact densities of e-p correlation function for $r_{\mathrm{s}}>4$ have been removed. This was done by introducing the appropriate form for electron-electron and electron-positron parts of the Jastrow factor. In particular, it is evident how important is the correction of the e-e part of the Jastrow factor. The 3-body correlations are also meaningful. Their contribution to the correlation function becomes considerable for $r_{\mathrm{s}}>5$, and rises for smaller electron densities, e.g. for $r_{\mathrm{s}}>8$ it exceeds $\approx 20 \%$ of the whole value of the contact density.

The new trial function can be used in diffusion Monte Carlo calculations, accelerating much the convergence of this method. The method can also serve as an additional tool for verification the existing many-body theories. Using the proposed MC formula for momentum dependent enhancement factors one can try to check e.g. the influence of Daniel-Vosko [24] effect on the enhancement near the $k_{\mathrm{F}}$ point (cf. [25]). In general, the new results will be useful in the next studies of positron annihilation in inhomogeneous media. Some values obtained from this "computational experiment" may become the benchmark points for DFT theories using LDA (or other approximations).

\section{Acknowledgments}

The author gratefully acknowledges several useful discussions with Dr. Ari Harju and Prof. Henryk Stachowiak. 


\section{References}

[1] H. Stachowiak, E. Boroński, http://aps.arxiv.org/abs/cond-mat/0402478, to be published.

[2] H. Stachowiak, E. Boroński, A.A. Saad, in: Proc. 33 Polish Seminar on Positron Annihilation (PSPA-33), Turawa (Poland) 2001, Ed. K. Jerie, University of Opole, University of Wrocław, Opole 2001, p. 107.

[3] J. Arponen, E. Pajanne, Ann. Phys. (New York) 121, 343 (1979).

[4] H. Stachowiak, Phys. Rev. B 41, 12522 (1990).

[5] A. Rubaszek, H. Stachowiak, Phys. Rev. B 38, 3846 (1988).

[6] V. Apaja, S. Denk, E. Krotscheck, Phys. Rev. B 68, 195118 (2003).

[7] L. Lantto, Phys. Rev. B 36, 5160 (1987).

[8] E. Boroński, R.M. Nieminen, Phys. Rev. B 34, 3820 (1986).

[9] D.M. Ceperley, M.H. Kalos, in: Monte Carlo Methods in Statistical Physics, Ed. K. Binder, Springer-Verlag, 1979.

[10] C.J. Umrigar, K.J. Runge, M.P. Nightingale, in: Monte Carlo Methods in Theoretical Physics, Eds. S. Caracoiolo, A. Fabrocini, Editrice, Pisa 1990.

[11] G. Ortiz, Ph.D. thesis, Ecole Polytechnique Fédérale de Lausanne, 1992, unpublished.

[12] L. Gilgien, Ph.D. thesis, Université de Genève, 1997, unpublished.

[13] L.M. Fraser, Ph.D. thesis, The Blackett Laboratory, University of London, 1995 (www.sst.ph.ic.ac.uk/research/theses/L.M.Fraser.pdf).

[14] E. Boroński, S. Siljamäki, in: Proc. 34 Polish Seminar on Positron Annihilation (PSPA-34), Turawa (Poland) 2002, Ed. K. Jerie, University of Opole, University of Wrocław, Opole 2002, p. 5.

[15] Xi Lin, H. Zhang, A.M. Rappe, J. Chem. Phys. 112, 2650 (2000).

[16] A. Harju, B. Barbiellini, S. Siljamäki, R.M. Nieminen, G. Ortiz, Phys. Rev. Lett. 79, 1173 (1997).

[17] G. Ortiz, P. Ballone, Phys. Rev. B 50, 1391 (1994).

[18] T. Kato, Commun. Pure Appl. Math. 10, 151 (1957).

[19] W.H. Young, Phys. Rev. 129, 2019 (1963).

[20] C.H. Leung, M.J. Stott, C.O. Almblath, Phys. Lett. A 57, 26 (1976).

[21] G.G. Ryzhikh, J. Mitroy, J. Phys. B 32, 4051 (1999).

[22] S. Kahana, Phys. Rev. 129, 1622 (1963).

[23] J. Arponen, E. Pajanne, J. Phys. C 12, 3013 (1979).

[24] E. Daniel, S. Vosko, Phys. Rev. 120, 2041 (1960).

[25] J.P. Carbotte, S. Kahana, Phys. Rev. A 139, 213 (1965). 